\title{
An-jun-ning, a traditional herbal formula, attenuates spontaneous withdrawal symptoms via modulation of the dopamine system in morphine-dependent rats
}

\author{
Jin-Long Gao', Shao-Ang Tu', Jia Liu', Jin-Ming Zhang ${ }^{2}$, Yiyun Huang ${ }^{3}$, Mei Han $^{1 *}$ and Jian-Hui Liang ${ }^{4^{*}}$
}

\begin{abstract}
Background: The dopamine system, which is involved in drug dependence, can be damaged by opioid abuse. However, current clinical medicines cannot reverse these damages in the brain, which are believed to be a key reason for the high relapse rate after abstinence treatment. This study aimed to investigate the effects of An-jun-ning (AJN), a commercial traditional Chinese medicine formula used for the treatment of opioid addiction, on the dopamine system in morphine-dependent rats and to explore the possible mechanism underlying its therapeutic effects.
\end{abstract}

Methods: The morphine dependence model was obtained through injections of morphine at increasing doses for 8 days. The AJN pre-treatment group was administered AJN 30 min before each morphine administration, and the AJN post-treatment groups were treated with AJN for 10 days after withdrawal. Spontaneous withdrawal symptoms (wet dog shakes, and episodes of writhing) were observed after withdrawal. Autoradiography study and/or immunohistochemical staining were used to examine the levels of dopamine transporter (DAT), dopamine $D_{2}$ receptor $\left(D_{2} R\right)$ and tyrosine hydroxylase $(T H)$.

Results: (1) Pre-treatment with AJN attenuates wet dog shakes and episodes of writhing to approximately 50\% or less of those observed in the morphine group $(p<0.01)$. (2) AJN post-treatment dose-dependently reduced the number of wet dog shakes $(p<0.01)$, and the episodes of writhing $(p<0.01)$. (3) Pre-treatment with AJN effectively interdicted the morphine-induced decreases in the levels of DAT, $D_{2} R$, and TH in the striatum $(p<0.01)$ such that they remained at nearly normal levels. (4) Post-treatment with AJN restored DAT and $D_{2} R$ to the normal levels ( $p<0.01$ ) and the level of TH to $87 \%$ of normal in the striatum.

Conclusions: AJN can effectively alleviate opioid withdrawal symptoms and preserve or restore the DAT, $D_{2} R$, and $\mathrm{TH}$ levels in the striatum. The mechanism underlying the effect of AJN on withdrawal symptoms may be related to the modulation of the dopamine system by AJN. These results suggest that AJN may help to prevent relapse in opioid dependence treatment.

Keywords: Morphine dependence, Dopamine transporter, Dopamine $D_{2}$ receptor, Tyrosine hydroxylase, An-jun-ning

\footnotetext{
* Correspondence: hanmei@bnu.edu.cn; liangjh@bjmu.edu.cn

'Key Laboratory of Radiopharmaceuticals, Ministry of Education, Department

of Chemistry, Beijing Normal University, Beijing 100875, China

${ }^{4}$ Department of Neuropharmacology, National Institute on Drug

Dependence, Peking University, Beijing 100191, China

Full list of author information is available at the end of the article
} 


\section{Background}

Opioid abuse and dependence is a chronic brain disorder and imposes severe medical and economic burdens on individuals and society. The United Nations Office on Drug and Crime estimates that 12 to 21 million people abuse opiates worldwide [1]. It has been reported that approximately $50 \%$ of patients experienced relapse behavior within a few days after completing treatment in a hospital [2]. Another follow-up investigation found that even when presenting to buprenorphine treatment, youth with opioid use within the past 30 days were less likely to avoid relapse at week 12 of treatment [3]. At present, there are some first-line medicines for the treatment of opioid addiction that effectively alleviate the opioid withdrawal symptoms. These include methadone, buprenorphine, naloxone, naltrexone, lofexidine and clonidine. But most of these drugs have undesirable side effects, such as abuse potential and high relapse rate and cannot effectively reverse the adaptive neurobiological changes. Therefore, more effective and safer approaches for the treatment of opioid addiction are urgently needed.

Traditional Chinese medicines (TCMs) have long been used to treat opioid addiction, and many prescriptions were proved to have therapeutic efficacy [4,5]. An-junning (AJN) is one of the TCMs approved by the China Food and Drug Administration for treatment of opioid addiction. Clinical studies have demonstrated that AJN is safe and well-tolerated by patients [6], and that it effectively alleviates the protracted withdrawal symptoms in heroin users $[7,8]$. Further, AJN has been reported to attenuate the impairments in tyrosine hydroxylase $(\mathrm{TH})$ and glial fibrillary acidic protein in the ventral tegmental area, thus potentially implicating the dopamine (DA) system in its therapeutic efficacy [9]. However, the exact mechanism underlying the effects of AJN has yet to be fully elucidated.

The DA system is believed to play an important role in addictive behaviors, including opioid addiction [10-12]. Long-term opioid abuse results in adaptive neurobiological changes in the brain, particularly in the DA system. For example, opioid abuse has been shown to be associated with decreased densities of dopamine transporters (DAT) and dopamine $\mathrm{D}_{2}$ receptors $\left(\mathrm{D}_{2} \mathrm{R}\right)$ in both animals and humans, and reduced TH activity in rats [13-17]. Current treatments for opioid addiction cannot reverse these neurobiological alterations, and can even cause exacerbation in some cases, resulting in high relapse rate after detoxification [18]. These neurobiological changes are therefore believed to be the key reason for the difficulties associated with abstinence treatment.

In this study, we examined the effects of AJN on the protracted withdrawal symptoms in morphine-dependent rats and sought to determine whether the morphine- induced decreases in DAT, $\mathrm{D}_{2} \mathrm{R}$ and $\mathrm{TH}$ can be interdicted (pre-treatment) or alleviated (post-treatment) by AJN. In another word, we attempted to investigate the hypothesis that modulation of the dopamine system by AJN is a possible mechanism of its therapeutic action.

\section{Methods}

\section{Animals}

Male Wistar rats weighing 180-220 g (Academy of Military Medical Science, Beijing, China) at the beginning of the experiment were used. The rats were housed in groups of five in a room with constant temperature $\left(25^{\circ} \mathrm{C}\right)$ and humidity (70\%) and a $12 \mathrm{~h}$ light/12 h dark cycle (08:0020:00 light on), with free access to food and water. Animals were maintained according to the international guidelines for the care and use of laboratory animals, and all experimental procedures involving animals were approved by the Ethics Committee of Beijing Normal University (BNU/EC/01/2011).

\section{Drugs and reagents}

AJN was provided by Taier Company (Hunan, China). Morphine hydrochloride was purchased from Qinghai Pharmaceutical Co. (China). (S)- $N$-((1-ethyl-2-pyrrolidinyl) methyl)-2-hydroxy-6-methoxy-3-(trimethylstannyl) benzamide (TBZM), and (1R,2S,3S,5S)-methyl 8-methyl-3-(4-(trimethylstannyl) phenyl)-8-azabicyclo [3.2.1] octane-2-carboxylate (trimethylstannyl- $\beta-C T$, or TMS- $\beta-C T$ ) were purchased from Huayi Isotope $\mathrm{Co}$. (Toronto, ON, Canada). $\mathrm{Na}^{125} \mathrm{I}$ (specific activity $>2200 \mathrm{Ci} / \mathrm{mmol}$ ) was purchased from Perkin Elmer (Boston, MA, USA). $\left[{ }^{125} \mathrm{I}\right]-\mathrm{IBZM}$ and $\left[{ }^{125} \mathrm{I}\right]-\beta$-CIT were prepared as described by Kung et al., and Toyama et al. [19,20].

\section{Morphine dependence model and AJN treatment}

The rats were divided into six groups: control group, morphine group, AJN pre-treatment group $(0.555 \mathrm{~g} / \mathrm{kg})$, and three AJN post-treatment groups: low dose (AJN-L, $0.185 \mathrm{~g} / \mathrm{kg}$ ), medium dose (AJN-M, $0.555 \mathrm{~g} / \mathrm{kg}$ ), and high dose (AJN-H, $1.851 \mathrm{~g} / \mathrm{kg}$ ). The rats in the morphine, AJN pre-treatment and AJN post-treatment groups were administered morphine via intraperitoneal injection twice daily (09:00 and 15:00) for eight days in a volume of $1 \mathrm{~mL} / \mathrm{kg}$ body weight, with a gradually increasing dose $(10,10,15,15,15,20,20$, and $20 \mathrm{mg} / \mathrm{kg}$ per injection on each day) [21,22]. The control animals received $0.9 \%$ saline in the same volume. AJN was dissolved in deionized water and intragastrically administered to the rats in the AJN pre-treatment group at a dose of $0.555 \mathrm{~g} / \mathrm{kg}$ ( $1 \mathrm{~mL} / \mathrm{kg}$ of body weight) $30 \mathrm{~min}$ before each morphine injection. After morphine administrations, the rats in the AJN post-treatment groups were administered AJN intragastrically once daily at a dose of 0.185 , 0.555 or $1.851 \mathrm{~g} / \mathrm{kg}$ ( $1 \mathrm{~mL} / \mathrm{kg}$ of body weight) for 10 days, whereas the other groups received the same volume of 
vehicle (saline). The AJN dosages were converted from those used clinically.

\section{Behavior observation}

At 10:00 a.m. on days one, five, and ten after the withdrawal of morphine, the animals were placed individually into Plexiglas cages and observed for signs of spontaneous withdrawal. Following a 5 min acclimation in the cages, the number of wet dog shakes and writhing episodes were monitored during a $30 \mathrm{~min}$ period [23]. Three observers blind to the groups completed the observation and score independently. Scores were averaged for each behavior test.

\section{Tissue preparation}

After the final behavioral observation, all the rats were sacrificed by decapitation. Rat brains were rapidly removed and stored at $-80^{\circ} \mathrm{C}$ until use. The brains were then cut into $18 \mu \mathrm{m}$ coronal slices with a cryostat (CM1900, Leica, Germany) at $-20^{\circ} \mathrm{C}$.

\section{Immunohistochemical staining}

Immunohistochemical staining to determine levels of DAT and $\mathrm{D}_{2} \mathrm{R}$ proteins and TH activity was conducted with striatal slices. To prepare for staining, the slices were prewashed in PBS (0.01 M, pH 7.4) three times for 5 min each, then sequentially treated with $0.2 \%$ Triton X-100 in PBS for 5 min and with $0.3 \% \mathrm{H}_{2} \mathrm{O}_{2}$ in PBS for $10 \mathrm{~min}$, and washed in PBS three times for $5 \mathrm{~min}$ each, all at room temperature. Slices were initially incubated with $10 \%$ normal goat serum for 10 min (or normal donkey serum for $\mathrm{TH}$ measurement), then incubated for $20 \mathrm{~h}$ at $4^{\circ} \mathrm{C}$ with the primary antibodies $\left(\mathrm{D}_{2} \mathrm{R}\right.$ antibody AB5084P, Millipore, CA, USA, 1:200 dilution; DAT antibody MAB369, Millipore, CA, USA, 1:100 dilution; TH antibody T1299, Sigma, CA, USA, 1:10,000 dilution), washed three times in PBS, and incubated for $60 \mathrm{~min}$ at $37^{\circ} \mathrm{C}$ with the secondary antibodies (anti-rabbit antibody PV-6001, ZSGB-BIO, CA, USA; anti-rat antibody ZB2307, ZSGB-BIO, CA, USA; anti-mouse antibody PK4002, Vector, CA, USA). Slices were then washed five times for 3 min each in PBS, and incubated in $100 \mu \mathrm{L}$ of 3,3' -diaminobenzidine tertrahydrochloride (DAB) for $3 \mathrm{~min}$. The slices immunostained for $\mathrm{TH}$ were incubated in the $\mathrm{ABC}$ (VECTASTAIN ABC kit, ZSGB-BIO, Beijing, China) reagent for $30 \mathrm{~min}$ at $37^{\circ} \mathrm{C}$ and washed five times for $3 \mathrm{~min}$ each in PBS prior to the DAB treatment. Final wash of the slices was done in distilled water.

\section{Autoradiography experiments}

The striatal slices were washed for $20 \mathrm{~min}$ at room temperature in $50 \mathrm{mM}$ Tris buffer $(\mathrm{pH} 7.4$, containing $120 \mathrm{mM} \mathrm{NaCl}, 5 \mathrm{mM} \mathrm{KCl}, 2 \mathrm{mM} \mathrm{CaCl}$, and $1 \mathrm{mM}$ $\mathrm{MgCl}_{2}$ ) for $\mathrm{D}_{2} \mathrm{R}$ autoradiography, and in $50 \mathrm{mM}$ Tris buffer ( $\mathrm{pH} 7.4$, containing $120 \mathrm{mM} \mathrm{NaCl}$ and $5 \mathrm{mM}$ $\mathrm{KCl})$ for DAT autoradiography. The slices were then incubated for $60 \mathrm{~min}$ in the same buffer with $50 \mathrm{pM}$ $\left[{ }^{125} \mathrm{I}\right]-\mathrm{IBZM}$ for $\mathrm{D}_{2} \mathrm{R}$ labeling or $50 \mathrm{pM}\left[{ }^{125} \mathrm{I}\right]-\beta$-CIT in the presence of $1 \mathrm{mM}$ fluoxetine (serotonin antagonist, Sigma-Aldrich Co., USA) for DAT labeling. The nonspecific binding was determined in the adjacent slices in the presence of $10 \mu \mathrm{M}$ sulpiride $\left(\mathrm{D}_{2} \mathrm{R}\right.$ antagonist, SigmaAldrich Co., USA) for $\mathrm{D}_{2} \mathrm{R}$ or in the presence of $100 \mathrm{mM}$ nomifensine (DAT antagonist, Sigma-Aldrich Co., USA) and $100 \mathrm{mM}$ fluoxetine for DAT. After incubation, the slices were washed five times for 1 min each in ice-cold $50 \mathrm{mM}$ Tris buffer ( $\mathrm{pH}$ 7.4), rapidly dipped in deionized water and dried under a stream of cold, dry air.

The labeled slices were mounted and exposed to a super-sensitive phosphor screen (PerkinElmer, USA) at room temperature for $8 \mathrm{~h}$. Densitometry determinations were performed using a Cyclone Plus phosphor imager (PerkinElmer, USA) and analyzed using the Opti-Quant software (PerkinElmer, USA). The specific binding detected in each structure was quantified by subtracting the non-specific binding image from the total binding image. The results are shown as the ratio of specific binding relative to the control group.

\section{Statistical analysis}

All of the statistical analyses were conducted using SPSS software (version 20.0) with a type I error rate of $\alpha=$ 0.05 (two-tailed). Data are expressed as the mean \pm SD. The significance of the changes in the behavioral assays was determined using the Kruskal-Wallis test. If the difference was found to be significant using this test, the Mann-Whitney U-test was used to compare the control and experimental groups. To compare the values obtained from two groups, Student's t-test was performed.

\section{Results}

\section{Effects of AJN on wet dog shakes and episodes of writhing}

We chose to use the spontaneous withdrawal model in this study because its course more closely follows that observed in the clinic than other withdrawal models, such as the naloxone-precipitated withdrawal model. Spontaneous withdrawal from chronic morphine treatment only induced mild signs after withdrawal. Abstinence was commonly evidenced by wet dog shakes and episodes of writhing.

As shown in Figure 1 (A and B), one day after withdrawal, the rats treated with morphine experienced many wet dog shakes. The number of wet dog shakes in the morphine group was $5.6 \pm 1.3$, which is significantly higher than that observed in the control group $(0.8 \pm 0.6)$. AJN pre-treatment significantly reduced the number of wet 

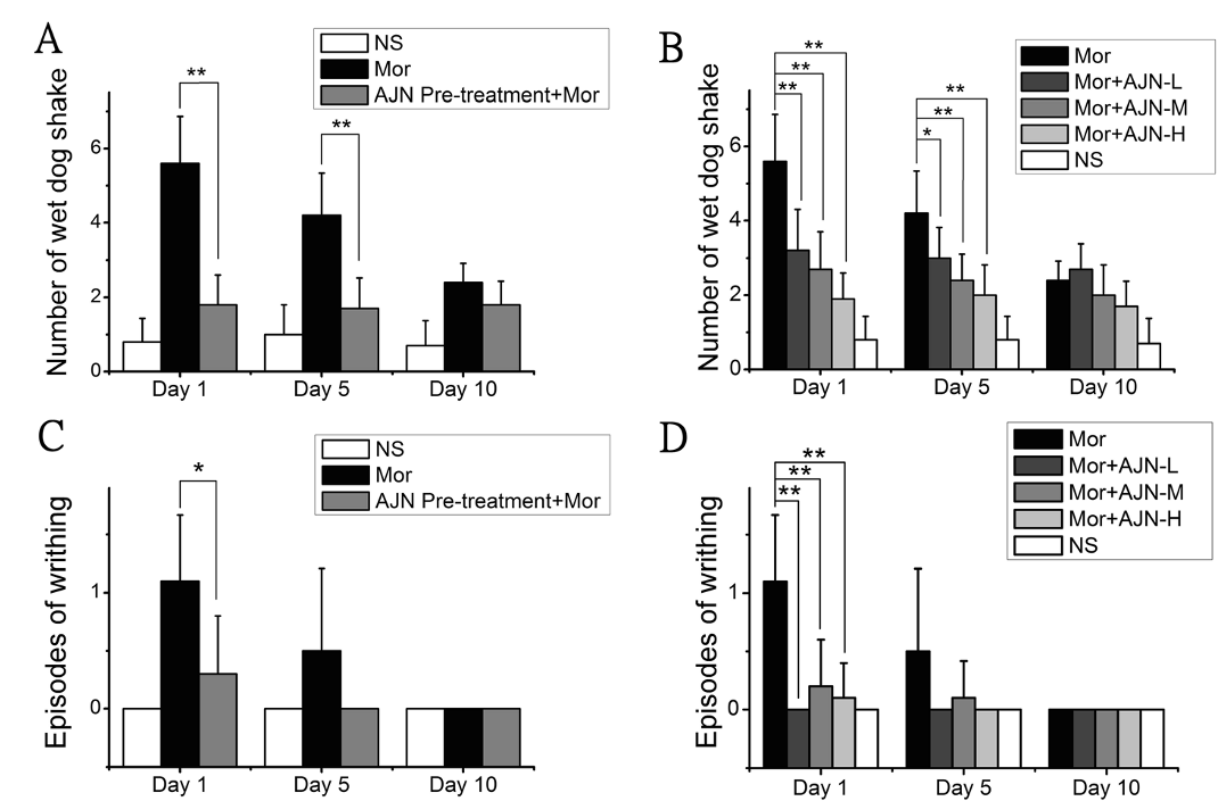

Figure 1 Effects of AJN on wet dog shakes and episodes of writhing after spontaneous withdrawal. A: AJN pre-treatment effectively holds wet dog shakes at a low level after 1, 5, 10 days of spontaneous withdrawal. B: AJN post-treatment alleviates wet dog shakes in a dose-dependent manner. C: AJN pre-treatment inhibits episodes of writhing after spontaneous withdrawal. D: AJN post-treatment attenuates episodes of writhing. NS: control group; Mor: morphine group. Data are expressed as the means $\pm S D\left({ }^{*} p<0.05,{ }^{* *} p<0.01\right.$ vs. morphine group; $\mathrm{n}=10 ;$ Kruskal-Wallis test and Mann-Whitney U-test).

dog shakes, to $1.8 \pm 0.8$. Additionally, AJN post-treatment alleviated wet dog shakes in a dose-dependent manner: animals treated with the low dose (AJN-L, $0.185 \mathrm{~g} / \mathrm{kg}$ ), medium dose (AJN-M, $0.555 \mathrm{~g} / \mathrm{kg}$ ) and high dose (AJN-H, $1.851 \mathrm{~g} / \mathrm{kg}$ ) AJN experienced 3.2 $\pm 1.1,2.7 \pm 1.0$, and $1.9 \pm$ 0.7 of wet dog shakes, respectively (Figure $1 \mathrm{~A}$ and B). Five days after withdrawal, the number of wet dog shakes in the morphine group was $4.2 \pm 1.1$, whereas animals in the AJN pre-treatment and post-treatment groups all exhibited lower numbers of wet dog shakes. Ten days later, at the end of the experiment, the rats in the morphine group still experienced a higher number of wet dog shakes than those in the control group, while those in the AJN-H post-treatment group experienced significantly lower number.

Episodes of writhing were observed after morphine withdrawal. The rats in the morphine group experienced $1.1 \pm 0.6$ episodes of writhing, whereas no rats in the control group showed this symptom. AJN pretreatment effectively reduced the episodes of writhing to a rate of $0.3 \pm 0.5(\mathrm{p}<0.05$, compared with the morphine group) (Figure 1C). Rats post-treated with AJN also experienced fewer episodes of writhing than those in the morphine group: AJN-L, AJN-M, and AJN-H treatment resulted in $0 \pm 0,0.2 \pm 0.4$ and $0.1 \pm 0.3$ episodes of writhing, respectively (Figure 1D). Five days after withdrawal, very few rats showed an episode of writhing (Figure $1 \mathrm{C}$ and $\mathrm{D}$ ).

\section{Effect of AJN on DAT, $D_{2} R$ and TH expression in the} striatum

\section{AJN effect on DAT}

Immunohistochemistry examination revealed that morphine administration in rats decreased the level of DAT to $87.2 \pm 4.1 \%$ of that observed in the control group $(\mathrm{p}<0.01$, compared with the control group, Figure 2A). AJN pretreatment completely inhibited this decrease and DAT expression was maintained $100.2 \pm 2.9 \%$ of the level observed in the control group, which was significantly higher than that in the morphine group. Additionally, groups post-treated with AJN-L, AJN-M, and AJN-H displayed DAT expression at $86.9 \pm 3.2 \%, 98.8 \pm 3.3 \%$ and $100.3 \pm 2.8 \%$ of the level observed in the control group, respectively, indicating that post-treatment with AJN-M and AJN-H normalized DAT expression in morphine dependent animals.

The immunohistochemistry results were confirmed by autoradiographic studies (morphine group: $87.8 \pm 4.8 \%$ of the control level; AJN pre-treatment: $104.1 \pm 4.7 \%$ of the control level; AJN-L: $88.9 \pm 4.6 \%$ of the control level; AJN-M: $97.2 \pm 6.7 \%$ of the control level; AJN-H: $99.5 \pm$ $8.4 \%$ of the control level) (Figure 2B).

\section{AJN effect on $D_{2} R$}

Immunohistochemical staining revealed that chronic morphine administration reduced $\mathrm{D}_{2} \mathrm{R}$ expression in the striatum to $83.2 \pm 4.0 \%$ of the level in the control group 


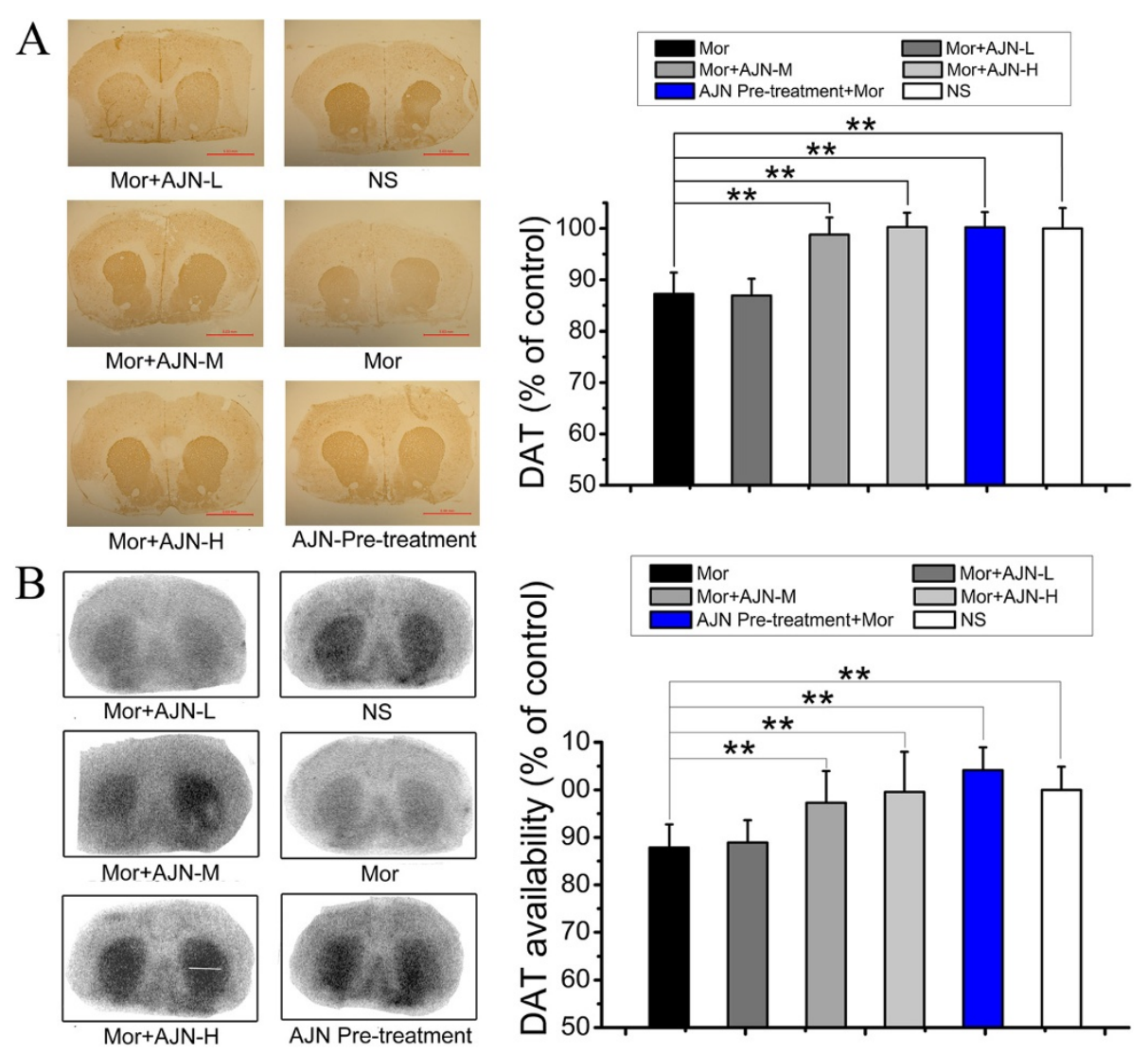

Figure 2 Effect of AJN pre-treatment on DAT by immunohistochemical staining and autoradiography. A: Representative staining pictures and effect of AJN on DAT by immunohistochemical staining. B: Representative autoradiograms and effect of AJN on DAT by autoradiography with $\left.{ }^{125} \mathrm{I}\right]-\beta-\mathrm{CIT}$. Results of the two methods are accordant with each other. AJN pre-treatment completely blocks morphine-induced DAT expression decrease, and AJN post-treatment effectively restores DAT expression. NS: control group; Mor: morphine group. Data are expressed as the means $\pm S D\left({ }^{*} p<0.05,{ }^{* *} p<0.01\right.$, vs. morphine group; $n=5$; Student's t-test).

( $\mathrm{p}<0.01$, compared with the control group, Figure 3A). AJN pre-treatment completely countered against this decrease and maintained DAT expression at $101.3 \pm 4.4 \%$ of the level observed in the control, which is significantly different from the level observed in the morphine group. Additionally, post-treatment with AJN-L, AJN-M, and AJN-H resulted in $\mathrm{D}_{2} \mathrm{R}$ expression at $83.1 \pm 3.5 \%, 96.7 \pm$ $2.7 \%$ and $100.3 \pm 1.7 \%$ of the level observed in the control group, indicating that both AJN-M and AJN-H posttreatments recovered $D_{2} R$ expression to the normal level. These findings by immunohistochemical staining were confirmed by autoradiography (morphine group: $78.2 \pm 6.0 \%$ of the control level; AJN pre-treatment: $102.1 \pm$ $3.6 \%$ of the control level; AJN-L: $80.1 \pm 5.9 \%$ of the control level; AJN-M: $95.0 \pm 6.9 \%$ of the control level; AJN-H: $102.2 \pm 9.9 \%$ of the control level) (Figure 3B).

\section{AJN effect on $\mathrm{TH}$ reactivity}

In the morphine group, $\mathrm{TH}$ reactivity in the striatum was reduced to $75.1 \pm 3.0 \%$ of the level in the control group $(\mathrm{p}<0.01$, compared with the control group, Figure 4).
AJN pre-treatment completely inhibited this decrease and maintained $\mathrm{TH}$ expression at $101.3 \pm 4.4 \%$ of the level in the control group, which is significantly different from that observed in the morphine group. Additionally, AJN post-treatment alleviated the decrease in $\mathrm{TH}$ expression in a dose-dependent manner: $\mathrm{TH}$ expression levels in the rats post-treated with $\mathrm{AJN}-\mathrm{L}, \mathrm{AJN}-\mathrm{M}$, and $\mathrm{AJN}-\mathrm{H}$ were $74.9 \pm 4.3 \%, 83.7 \pm 4.6 \%$, and $87.0 \pm 2.8 \%$, respectively, of the control level.

\section{Discussion}

This study provides the first preclinical investigation on the modulation of the dopamine system as a mechanism underlying the therapeutic action of the traditional Chinese medicine formula An-jun-ning in alleviating spontaneous withdrawal symptoms in opioid dependence. Results from the present study demonstrated that AJN effectively alleviated the morphine withdrawal symptoms. Immunohistochemical and autoradiographic studies indicated that pre-treatment with AJN inhibited the morphine-induced decreases in DAT, $\mathrm{D}_{2} \mathrm{R}$, and $\mathrm{TH}$ 
A

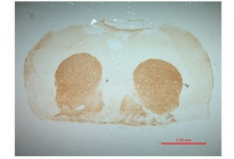

Mor+AJN-L

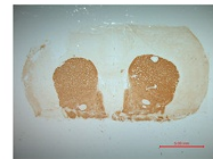

Mor+AJN-M

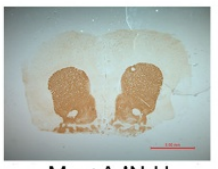

Mor+AJN-H

B

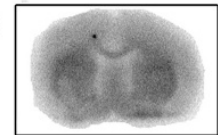

Mor+AJN-L

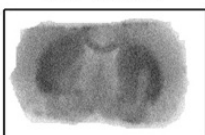

Mor+AJN-M

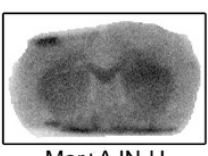

Mor+AJN-H

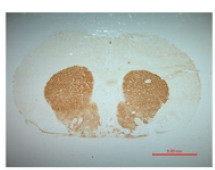

NS

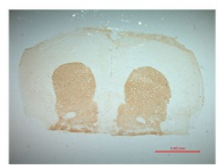

Mor

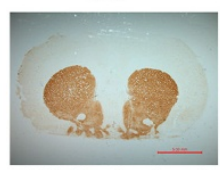

AJN Pre-treatment

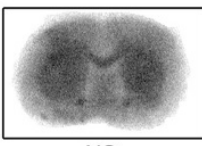

NS

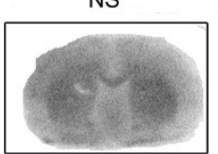

Mor

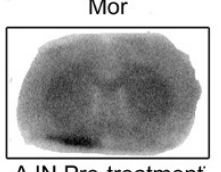

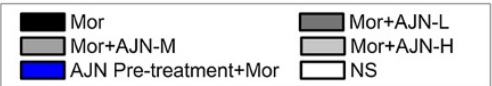

$\star \star$
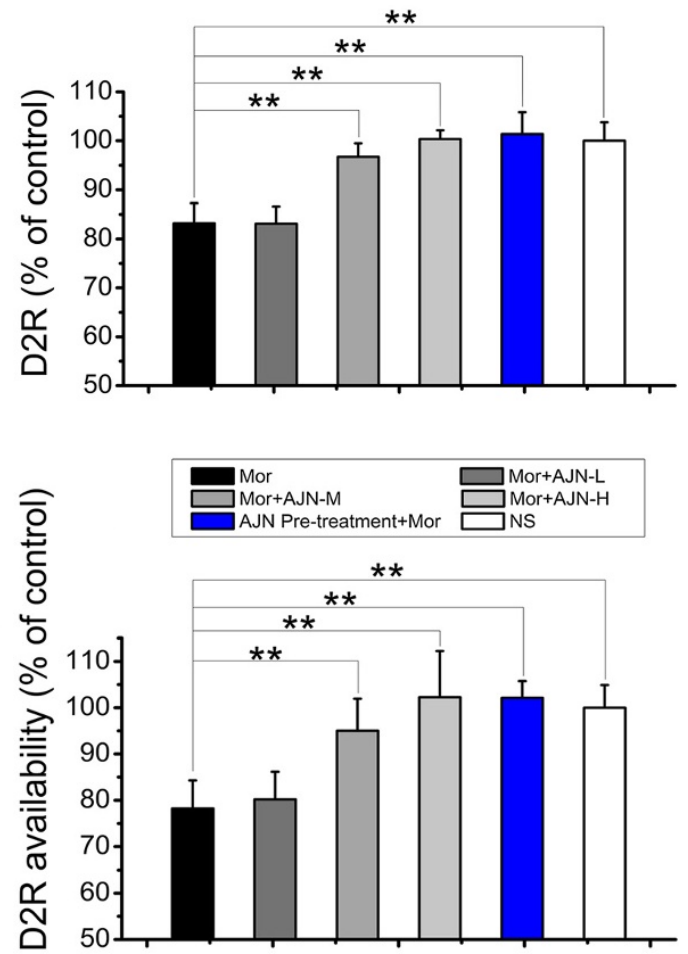

Figure 3 Effect of $\mathbf{A J N}$ on $\mathbf{D}_{2} \mathbf{R}$ by immunohistochemical staining and autoradiography. A: Representative staining pictures and effect of AJN on $D_{2} R$ by immunohistochemical staining. B: Representative autoradiograms and effect of AJN on $D_{2} R$ by autoradiography with $\left[{ }^{125}\right.$ I]-IBZM. Morphine administration reduces $D_{2} R$ expression by about 20\%. AJN pre-treatment completely inhibits the morphine-induced $D_{2} R$ decrease. And AJN post-treatment alleviate the decrease in $D_{2} R$ expression in a dose-dependent manner. NS: control group; Mor: morphine group. Data are expressed as the means $\pm \mathrm{SD}\left({ }^{*} p<0.05,{ }^{* *} p<0.01\right.$, vs. morphine group; $n=5$; Student's t-test).

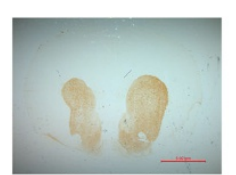

Mor+AJN-L

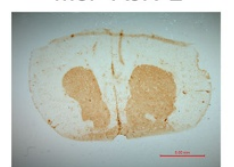

Mor+AJN-M

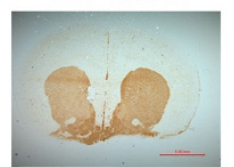

Mor+AJN-H

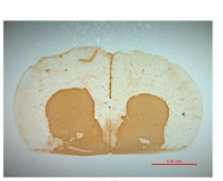

NS

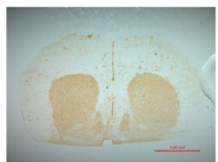

Mor

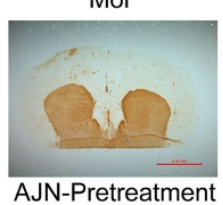

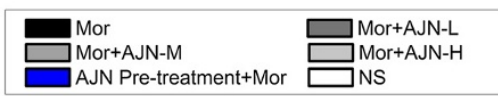

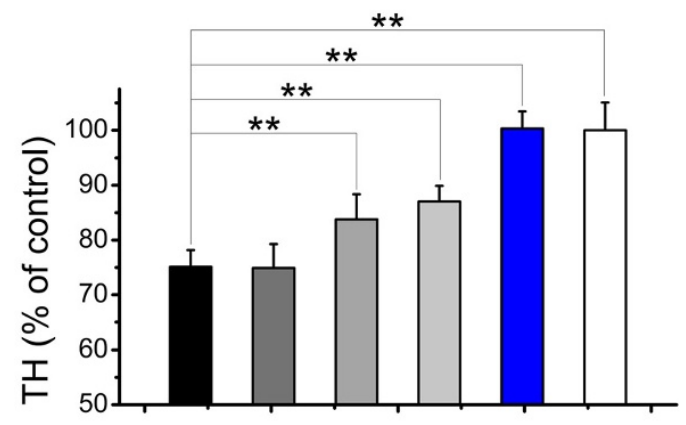

Figure 4 Effect of AJN on TH density by immunohistochemical staining. Representative staining pictures and effect of AJN on TH density by immunohistochemical staining. TH density in the striatum is decreased in the morphine group. Both AJN pre- and post-treatment effectively attenuates this decrease. NS: control group; Mor: morphine group. Data are expressed as the means \pm SD $\left({ }^{*} p<0.05,{ }^{* *} p<0.01, \mathrm{vs}\right.$. morphine group; $n=5$; Student's t-test). 
expression in the striatum, suggesting that a mechanism of action for AJN might be related to its modulation of the dopamine system. Additionally, post-treatment with medium or high dose of AJN normalized DAT and $\mathrm{D}_{2} \mathrm{R}$ expression in morphine dependent rats, which confirms that AJN can effectively act on the dopamine system. Taken together, these results provide support for the hypothesis that the mechanisms by which AJN mitigates morphine withdrawal symptoms involve modulation of the DA system.

The restoration of DA function is believed to be beneficial in the treatment of opioid addiction and can result in reduced relapse rate. There is considerable evidence for the dysregulation of DA system following repeated drug intake, and the changes observed persist throughout the early phases of abstinence. Chronic administration of morphine or heroin to rodents has been shown to cause decreases in striatal concentrations of synaptic DA, TH, DAT, and $\mathrm{D}_{2} \mathrm{R}$ [15,24-27]. Decreased levels of $\mathrm{DA}, \mathrm{DAT}$ and $\mathrm{D}_{2} \mathrm{R}$ have also been found in patients addicted to heroin [16-18]. Collectively, these findings are consistent with the hypothesis that an effective treatment for opioid addiction may be achieved if the neurologic impairments are alleviated and DA function is restored [28]. In this study, we examined the effects of pre- and post-treatment with AJN on striatal DAT, $D_{2} R$, and $\mathrm{TH}$ levels in a rat model of morphine-dependence. Our results demonstrated that 1) morphine dependence induced decreases in DAT, $\mathrm{D}_{2} \mathrm{R}$ and $\mathrm{TH}$ levels in the striatum; 2) these decreases were effectively inhibited by pre-treatment with $\mathrm{AJN}$; and 3) post-treatment with AJN acted against these decreases, with medium and high dose AJN post-treatment restoring DAT, $\mathrm{D}_{2} \mathrm{R}$ in the morphine model rats to the normal levels. Taken together, results from our present study support the hypothesis that the dopamine system plays an important role in the therapeutic mechanism of AJN and thus provide a novel strategy for the treatment of opioid addiction.

Based on previous studies of Rhizoma Corydalis, which is one of the major ingredients in $\mathrm{AJN}$, we postulate that the effect of AJN on the dopamine system might be partly related to the compounds found in Rhizoma Corydalis [29]. Previous studies showed that $l$-tetrahydropalmatine ( $l$-THP), the primary active component in Rhizoma Corydalis, attenuated the conditioned place preference in morphine-dependent rats, and acted on the $D_{2} R$ in the striatum to enhance endogenous opioid peptide function, and hence accelerated the functional recovery of the dopamine system [30-32]. These studies provided support for the hypothesis that $l$-THP might play an important role in the therapeutic effects of AJN on the dopamine system. On the other hand, since AJN is a traditional Chinese medicine formula with multiple other components in addition to Rhizoma Corydalis (Table 1), it is
Table 1 Composition of AJN

\begin{tabular}{ll}
\hline Scientific name & Amount (\%) \\
\hline Rhizoma Corydalis & 39.78 \\
Radix Paeoniae Alba & 15.75 \\
Glycyrrhiza uralensis Fisch & 2.39 \\
Syringa Linn & 2.39 \\
Houttuynia cordata Thunb & 11.93 \\
Terminalia chebula Retz & 11.93 \\
Radix Cynanchi Paniculati & 3.98 \\
Alpinia oxyphylla & 3.98 \\
Arisaema cum bile & 7.87 \\
Total amount & 100.00 \\
\hline
\end{tabular}

reasonable to contemplate that, besides Rhizoma Corydalis and $l$-THP, other components and ingredients may also contribute to its efficacy in alleviating morphine withdrawal symptoms, and its protective/restorative effects on dopamine function. Much work remains to be done to fully elucidate the pharmacological targets involved in the therapeutic action of AJN.

Nonetheless, results from the present study provide the first evidence that AJN may exert its therapeutic action for the treatment of opioid dependence through modulation of the dopamine system, as we demonstrated that AJN not only protects the DA system from the deleterious effects of morphine (through pretreatment studies), but also counteracts against these effects (via post-treatment studies), both of which involve the maintenance of DAT, $\mathrm{D}_{2} \mathrm{R}$ and $\mathrm{TH}$ at normal levels.

Availability of DAT and $\mathrm{D}_{2} \mathrm{R}$ can be detected clinically through molecular imaging methods, such as positron emission tomography (PET) and single photon emission computed tomography (SPECT) $[14,16]$. In the current study we examined DAT and $\mathrm{D}_{2} \mathrm{R}$ levels via autoradiography, which is based on the same principles as PET and SPECT imaging. Results from autoradiography studies are corroborated by and consistent with those obtained through immunohistochemical staining, which is an accepted protein detection technique. Together, these results provide strong preclinical evidence for the ability of AJN to simultaneously counter against the morphineinduced decreases in the levels of DAT, $\mathrm{D}_{2} \mathrm{R}$, and $\mathrm{TH}$ in the striatum and to offer relief from morphine withdrawal symptoms. Hence, our study offers new insights into the mechanism of AJN therapeutic action and implies the modulation of dopamine system as an effective avenue for the treatment of opioid addiction. Future clinical studies using PET or SPECT imaging will serve to verify these findings in patients and advance our understanding and development of effective therapeutic interventions in opioid dependence. 


\section{Conclusions}

In this report we demonstrate that the traditional Chinese medicine formula An-jun-ning effectively alleviates the spontaneous morphine withdrawal symptoms. Further, AJN is shown to normalize DAT, $\mathrm{D}_{2} \mathrm{R}$ and TH levels in the striatum of morphine dependent animals. Hence, this study offers the first demonstration of AJN's effects on the dopamine system, and thus provides insights into the clinical benefits of AJN treatment for opioid addiction. Further studies are warranted to examine AJN therapeutic effects on the dopamine system in the clinic.

\section{Abbreviations}

AJN: An-jun-ning (a traditional Chinese medicine); DA: Dopamine; DAT: Dopamine transporter; $D_{2} R$ : Dopamine $D_{2}$ receptor; $T H$ : Tyrosine hydroxylase; TCM: Traditional Chinese medicine; I-THP: I-tetrahydropalmatine; PET: Positron emission tomography; SPECT: Single photon emission computed tomography.

\section{Competing interests}

The authors declare no competing financial interests. The Taier company, manufacturer of AJN, played no role in this study beyond providing the herbal formula used.

\section{Authors' contributions}

$J \mathrm{LG}$ designed and carried out the behavior tests, prepared the radiotracers, established assay conditions and helped draft the manuscript. SAT carried out the autoradiography and immunohistochemical staining experiments, and drafted the manuscript. $J \mathrm{~L}$ participated in tissue preparation and statistical analysis. JMZ and YH provided expertise in autoradiography and helped in data analysis, result interpretation, and manuscript preparation. JHL and $\mathrm{MH}$ designed and coordinated the study, and finalized the manuscript. All authors read and approved the final manuscript.

\section{Acknowledgements}

This study was supported by the National Key Technology R \& D Program (2008BAl49B04), the China National Science Foundation (81173139) and the Major Research Plan of NSFC (21233003). We thank the Taier Company for providing AJN used in this study.

\section{Author details}

${ }^{1}$ Key Laboratory of Radiopharmaceuticals, Ministry of Education, Department of Chemistry, Beijing Normal University, Beijing 100875, China. ${ }^{2}$ Chinese PLA General Hospital, Beijing, China. ${ }^{3}$ Yale PET Center, Department of Diagnostic Radiology, Yale University School of Medicine, New Haven, CT, USA. ${ }^{4}$ Department of Neuropharmacology, National Institute on Drug Dependence, Peking University, Beijing 100191, China.

Received: 12 November 2013 Accepted: 16 July 2014 Published: 19 August 2014

\section{References}

1. UNDOC: World Drug Report 2011. New York: United Nations; 2011.

2. Gossop M, Bradley B, Phillips GT: An investigation of withdrawal symptoms shown by opiate addicts during and subsequent to a 21-day in-patient methadone detoxification procedure. Addict Behav 1987, 12(1):1-6.

3. Subramaniam GA, Warden D, Minhajuddin A, Fishman MJ, Stitzer ML, Adinoff B, Trivedi M, Weiss R, Potter J, Poole SA: Predictors of abstinence: National Institute of Drug Abuse multisite buprenorphine/naloxone treatment trial in opioid-dependent youth. J Am Acad Child Adolesc Psychiatry 2011, 50(11):1120-1128.

4. Mattioli L, Titomanlio F, Perfumi M: Effects of a Rhodiola rosea L. extract on the acquisition, expression, extinction, and reinstatement of morphine-induced conditioned place preference in mice. Psychopharmacology (Berl) 2012, 221(2):183-193.
5. Shi J, Liu YL, Fang YX, Xu GZ, Zhai HF, Lu L: Traditional Chinese medicine in treatment of opiate addiction. Acta Pharmacol Sin 2006, 27(10):1303-1308.

6. Li J, Li XL, Peng ZG, Kuang WH, Huang MS: The study of suffertibility of Anjunning for volunteer. West Chin Med J 2000, 15(1):102-103.

7. Shen HX, Hao W, Liu TQ, Zhang RL, Su ZH, Liu KJ: A controlled study on clinical efficacy of Chinese herbal compounds, Anjunning and Kanfuxin on alleviating opioid protracted abstinent symptoms. Chin J Clin Psychol 2004, 12(1):38-40.

8. Xu BS, Wang ZQ, LV QL, Jin J, Shuai YL, Wang YF: A controlled study of An-jun-ning, a traditional Chinese medicine for the treatment of protracted withdrawal symptoms. Chin J Drug Depend 2003, 12(4):276-279.

9. Shen HX, Hao W, Cai Y, Xiang X, Wang X: The effects of Anjunning on tyrosine hydroxylase (TH) and glial fibrillary acidi cprotein (GFAP) in VTA of morphine dependent SD rats. Chin J Behav Med Sci 2006, 15(5):423-426.

10. Di Chiara G, Bassareo V: Reward system and addiction: what dopamine does and doesn't do. Curr Opin Pharmacol 2007, 7(1):69-76.

11. Melis M, Spiga S, Diana M: The dopamine hypothesis of drug addiction: hypodopaminergic state. Int Rev Neurobiol 2005, 63:101-154.

12. Wu G, Huang W, Zhang H, Li Q, Zhou J, Shu H: Inhibitory effects of processed Aconiti tuber on morphine-induced conditioned place preference in rats. J Ethnopharmacol 2011, 136(1):254-259.

13. Kish SJ, Kalasinsky KS, Derkach P, Schmunk GA, Guttman M, Ang L, Adams V, Furukawa Y, Haycock JW: Striatal dopaminergic and serotonergic markers in human heroin users. Neuropsychopharmacology 2001, 24(5):561-567.

14. Martinez D, Saccone PA, Liu F, Slifstein M, Orlowska D, Grassetti A, Cook S, Broft A, Van Heertum R, Comer SD: Deficits in dopamine $D_{2}$ receptors and presynaptic dopamine in heroin dependence: commonalities and differences with other types of addiction. Biol Psychiatry 2012, 71(3):192-198

15. Simantov R: Chronic morphine alters dopamine transporter density in the rat brain: possible role in the mechanism of drug addiction. Neurosci Lett 1993, 163(2):121-124.

16. Liu Y, Han M, Liu X, Deng Y, Li Y, Yuan J: Dopamine transporter availability in herion-dependent subjects and controls: longitudinal changes during abstinence and the effects of Jitai tablets treatment. Psychopharmacology (Berl) 2013, 230(2):235-244.

17. Yeh TL, Chen KC, Lin SH, Lee H, Chen PS, Yao WJ, Lee SY, Yang YK, Lu RB, Liao $\mathrm{MH}$ : Availability of dopamine and serotonin transporters in opioid-dependent users-a two-isotope SPECT study. Psychopharmacology (Berl) 2012, 220(1):55-64

18. Shi J, Zhao LY, Copersino ML, Fang YX, Chen YM, Tian JH, Deng YP, Shuai $Y L$, Jin J, Lu L: PET imaging of dopamine transporter and drug craving during methadone maintenance treatment and after prolonged abstinence in heroin users. Eur J Pharmacol 2008, 579(1):160-166.

19. Kung MP, Liu BL, Yang YY, Billings JJ, Kung HF: A kit formulation for preparation of iodine-123-IBZM: a new CNS $D_{2}$ dopamine receptor imaging agent. J NuCl Med 1991, 32(2):339-342.

20. Toyama $H$, Ichise $M$, Ballinger JR, Fornazzari $L$, Kirsh JC: Dopamine $D_{2}$ receptor SPECT imaging: Basicin vivo characteristics and clinical applications of ${ }^{123}$ I-IBZM in humans. Ann Nucl Med 1993, 7(1):29-38.

21. Darvishzadeh-Mahani F, Esmaeili-Mahani S, Komeili G, Sheibani V, Zare L: Ginger ( Zingiber officinale Roscoe) prevents the development of morphine analgesic tolerance and physical dependence in rats. J Ethnopharmacol 2012, 141(3):901-907.

22. Fukunaga $Y$, Inoue $N$, Miyamoto $M$, Kishioka $S$, Yamamoto $H$ : Effects of peptidase inhibitors,[D-Ala ${ }^{2}$, Met ${ }^{5}$ ]-enkephalinamide and antiserum to methionine-enkephalin microinjected into the caudal periaqueductal gray on morphine withdrawal in rats. Jpn J Pharmacol 1998, 78(4):455-461.

23. Zharkovsky A, Tötterman A, Moisio J, Ahtee L: Concurrent nimodipine attenuates the withdrawal signs and the increase of cerebral dihydropyridine binding after chronic morphine treatment in rats. Naunyn Schmiedebergs Arch Pharmacol 1993, 347(5):483-486.

24. Acquas E, Carboni E, Di Chiara G: Profound depression of mesolimbic dopamine release after morphine withdrawal in dependent rats. Eur J Pharmacol 1991, 193(1):133-134.

25. Crippens D, Robinson TE: Withdrawal from morphine or amphetamine: different effects on dopamine in the ventral-medial striatum studied with microdialysis. Brain Res 1994, 650(1):56-62.

26. Self DW, McClenahan AW, Beitner-Johnson D, Terwilliger RZ, Nestler EJ: Biochemical adaptations in the mesolimbic dopamine system in response to heroin self-administration. Synapse 1995, 21(4):312-318. 
27. Wang GJ, Volkow ND, Fowler JS, Logan J, Abumrad NN, Hitzemann RJ, Pappas NS, Pascani K. Dopamine $D_{2}$ receptor availability in opiate-dependent subjects before and after naloxone-precipitated withdrawal. Neuropsychopharmacology 1997, 16(2):174-182

28. Leshner Al: Addiction is a brain disease, and it matters. Science 1997, 278(5335):45-47.

29. Lu L, Liu Y, Zhu W, Shi J, Liu Y, Ling W, Kosten TR: Traditional medicine in the treatment of drug addiction. Am J Drug Alcohol Abuse 2009, 35(1):1-11.

30. Yan Bo W, Yan Hua R, Ji Wang Z, Lan Z: Influence of I-tetrahydropalmatine on morphine-induced conditioned place preference. Chin Pharmacol Bull 2005, 21(12):1442.

31. Su HL, Zhu J, Chen YJ, Zhao N, Han W, Dang YH, Xu M, Chen T: Roles of levo-tetrahydropalmatine in modulating methamphetamine reward behavior. Physiol Behav 2013, 118:195-200.

32. Figueroa-Guzman Y, Mueller C, Vranjkovic O, Wisniewski S, Yang Z, Li S-J, Bohr C, Graf EN, Baker DA, Mantsch JR: Oral administration of levo-tetrahydropalmatine attenuates reinstatement of extinguished cocaine seeking by cocaine, stress or drug-associated cues in rats. Drug Alcohol Depend 2011, 116(1):72-79.

doi:10.1186/1472-6882-14-308

Cite this article as: Gao et al: An-jun-ning, a traditional herbal formula, attenuates spontaneous withdrawal symptoms via modulation of the dopamine system in morphine-dependent rats. BMC Complementary and Alternative Medicine 2014 14:308.

\section{Submit your next manuscript to BioMed Central and take full advantage of:}

- Convenient online submission

- Thorough peer review

- No space constraints or color figure charges

- Immediate publication on acceptance

- Inclusion in PubMed, CAS, Scopus and Google Scholar

- Research which is freely available for redistribution 